\title{
THE STRUCTURE OF HYPERGROUP MEASURE ALGEBRAS
}

\author{
W. CHRISTOPHER LANG
}

(Received 16 January 1987)

Communicated by J. F. Price

\begin{abstract}
A close analogue for some hypergroup measure algebras of the structure semigroup theorem of J. L. Taylor for convolution measure algebras is constructed: a structure semihypergroup representation is made for the hypergroup measure and its spectrum. This is done for those hypergroup measure algebras that satisfy a condition known as the structure-strong condition. This condition is that the norm-closure of the linear span of the spectrum of the hypergroup measure algebra is a commutative $B^{*}$-algebra. Then examples of hypergroups whose measure algebras satisfy this condition are given. They include the space of $B$-orbits of $G$, where $B$ is a finite solvable group of automorphisms on a locally compact abelian group $G$. (The hypergroup measure algebra may be identified with the algebra of $B$-invariant measures on $G$.) Other examples are the algebra of central measures on a compact, connected, semisimple Lie group, and the algebra of rotation invariant measures on the plane.
\end{abstract}

1980 Mathematics subject classification (Amer. Math. Soc.): 43 A 10.

Keywords and phrases: abstract harmonic analysis, measure algebras, hypergroups, structure semigroups.

\section{Introduction}

Our goal has been to duplicate, for hypergroup measure algebras, the program of Joseph L. Taylor. He developed his structure semigroup representation of commutative group measure algebras and successfully applied it to the study of the structure of those algebras. (See Taylor [17].) The results of Taylor

This paper is based on the doctoral dissertation I wrote under the direction of Dr. Kenneth Ross. I would like to express my gratitude for his help.

(C) 1989 Australian Mathematical Society 0263-6115/89 \$A2.00 + 0.00 
encompass essentially only commutative semigroup measure algebras. The hope is to extend his results to noncommutative algebras or algebras not arising from groups. Here, under the restricted hypothesis of the structure-strong condition, a close analogue to the structure semigroup representation (Taylor $[17,3.2 .3]$ ) is built, for certain hypergroup measure algebras. These measure algebras include the algebra of central measures on a compact connected semisimple Lie group, and the algebra of rotation invariant measures on the plane. These examples rely on the calculations of their spectra by D. Ragozin [11], [12]. A different class of examples is provided by the algebra of measures on a locally compact abelian group which are invariant under a finite solvable group of automorphisms.

Another approach to structural representations of hypergroup measure algebras is found in C. Dunkl [3]. His theorem [3, 1.5] resembles the "structure semihypergroup" Theorem 4.1 below; Dunkl requires a condition on the spectrum of the measure algebra different than the "structure-strong" condition introduced in Section 3 below. Dunkl gives a sharper condition [3, 3.1] which ensures that certain simple algebras satisfy the hypotheses of [3, Theorem 1.5], and gives examples, such as the hypergroup measure algebra arising from ultraspherical series, which satisfy the conditions. These results resemble Ragozin's results [11], [12]. Dunkl conjectured that the algebra of central measures on a compact simple Lie group also satisfies his condition [3, 3.1]. (As stated above, our "structure-strong" condition will be shown to hold for the algebra of central measures on a compact connected semisimple Lie group.)

The definition of hypergroup is given in Jewett [9], our basic reference on hypergroups (which he calls "convos"). Essentially, a hypergroup $H$ is a locally compact space with a binary operation $H \times H \rightarrow P M(H)$, where $P M(H)$ is the set of probability measures on $H$, satisfying certain "convolution properties" (see below). Examples are provided by many orbit spaces, for example, the orbits in the plane, of the group of all rotations about the origin, form a hypergroup. The hypergroup operation corresponds to the convolution of rotation-invariant measures on the plane. Another example is given by the algebra of central measures on a compact group (the underlying hypergroup is the space of inner automorphism orbits, that is, conjugacy classes.) (See Ross [14] for more information of an expository nature.)

Hypergroups as defined by Jewett have the following three important properties.

(a) $\int_{H} f d \mu * \nu=\int_{H} \int_{H}\left\{\int_{H} f d p_{x} * p_{y}\right\} d \mu(x) d \nu(y)$ for all $\mu, \nu \in M(H)$, $f \in C_{0}(H)$. We often write $f(x * y)$ for $\int_{H} f d p_{x} * p_{y}$, even though $x * y$ has no independent meaning, so we may write the above equation as $\int_{H} f d \mu * \nu=$ $\int_{H} \int_{H} f(x * y) d \mu(x) d \nu(y)$. 
(b) If $\mu, \nu \in M(H)$ are probability measures, then $\mu * \nu$ is a probability measure.

(c) $M(H)$ with $*$ is a Banach algebra.

These properties are the features of a hypergroup we will generally use. Normally, we will assume hypergroups to be commutative (that is, $M(H)$ is a commutative Banach algebra).

We set down some definitions and notation. Our principal references are Taylor [17], Jewett [9], and Rennison [13].

Let $A$ be a Banach algebra. Then let $A_{1}=\{a \in A:\|a\| \leq 1\}$. Let $A^{+}$ be the set of all positive elements of $A$. Let $A^{*}$ be the dual space of $A$ (all continuous linear functionals on $A$ ). Let $\Delta A$ be the spectrum of $A$ (all nonzero multiplicative linear functionals on $A$ ). Note $\Delta A \subseteq A^{*}$.

Let $X$ be locally compact and Hausdorff. Let $M(X)$ be the set of all finite regular complex Borel measures on $X$. We will denote by $p_{x}$ the point mass at $x \in X$. Let $C_{0}(X)$ be the space of all continuous functions which vanish at infinity (that is, for $\varepsilon>0$ there exists a compact $K \subseteq X$ such that $|f(x)|<\varepsilon$ for all $x \notin K)$. Let $C_{00}(X)$ be the space of all continuous functions with compact support. Then the Riesz representation theorem says that $C_{0}(X)^{*}=M(X)$ by the isometric isomorphism $\mu \rightarrow F_{\mu}: M(X) \rightarrow C_{0}(X)^{*}$, where $F_{\mu}(f)=\int_{x} f d \mu$ for all $f \in C_{0}(X)$.

We now consider certain products of $M(H)^{*}$ and $M(H)^{* *}$ for a hypergroup $H$. Suppose $H$ is a commutative hypergroup. A fortiori $H$ is a locally compact Hausdorff space. Then $M(H)$ is an $L$-space (see Taylor [17, 2.1.1]), and hence $M(H)^{*}$ is a commutative unital $B^{*}$-algebra. This follows from Taylor $[17,2.3]$; the product given on $M(H)^{* *}$ is the product of Sreider generalized functions. (For $f \in M(H)^{*}$, for each $\mu \in M(H)$ let $f_{\mu} \in L^{\infty}(H, \mu)$ be the $\mu$ essentially bounded function that corresponds to $f$ restricted to $L^{1}(H, \mu)$. Then $\left\{f_{\mu}\right\}_{\mu \in M(H)}$ is the generalized function for $f$. So $f(\nu)=\int_{H} f_{\mu} d \nu$ for any $\nu \ll \mu$. Now consider $f, g \in M(H)^{*}$. They have generalized functions $\left\{f_{\mu}\right\},\left\{g_{\mu}\right\}$ respectively. Then $f g$ is the bounded linear functional whose generalized function is $\left\{f_{\mu} g_{\mu}\right\}$. That is, define $f g \in M(H)^{*}$ by $(f g)(\mu)=\int_{H} f_{\mu} g_{\mu} d \mu$ for all $\mu \in M(H)$. Also, the involution on $M(H)^{*}$ is defined by $f^{*}(\mu)=f(\bar{\mu})^{-}$for all $\mu \in M(H)$, $f \in M(H)^{*}$ (so the generalized function of $f^{*}$ is $\left\{\left(f_{\mu}\right)^{-}\right\}$. Here $\bar{\mu}$ is the usual involution of $M(H)$ (and the other superscript bars are complex convolution). The unit of $M(H)^{*}$ is $1(\mu)=\mu(H)=\int_{H} 1 d \mu$, so the generalized function is just the constant one function. That this is well defined and makes $M(H)^{*}$ a commutative unital $B^{*}$-algebra is Theorem 2.3.4 of Taylor [17].)

The Arens product on $M(H)^{* *}$ is defined in three stages (this is actually the right Arens product) as follows. 
(1) For $f \in M(H)^{*}$ and $\mu \in M(H)$ define $\langle f, \mu\rangle \in M(H)^{*}$ by $\langle f, \mu\rangle(\nu)=$ $f(\mu * \nu)$ for all $\nu \in M(H)$.

(2) For $F \in M(H)^{* *}$ and $f \in M(H)^{*}$ define $[F, f] \in M(H)^{*}$ by $[F, f](\mu)=$ $F(\langle f, \mu\rangle)$ for all $\mu \in M(H)$.

(3) For $F, G \in M(H)^{* *}$ define $F G \in M(H)^{* *}$ by $F G(f)=F([G, f])$ for all $f \in M(H)^{*}$.

The following lemma will be useful.

LEMMA 1.1. In any measure space $M(K)$, where $K$ is a locally compact Hausdorff space, a measure $\rho \in M(K)$ is positive if and only if $\int_{k} 1 d \rho=\|\rho\|$.

ProOF. Without loss of generality let $\|\rho\|=1$. Clearly $\rho \geq 0$ implies $\int_{k} 1 d \rho=\|\rho\|$. By polar decomposition, $d \rho=h d|\rho|$ where $|h| \equiv 1 \rho$-a.e. Suppose $h=\alpha+\beta i$ ( $\alpha, \beta$ real-valued). Then $1=\int_{k} 1 d \rho=\int_{k} \alpha d|\rho|$ so $\int_{k} \beta d|\rho|=0$ and $\int_{k} \alpha d|\rho|=1$. Since $|h| \equiv 1 \rho$-a.e., $-1 \leq \alpha \leq 1 \rho$-a.e. This forces $\alpha \equiv 1 \rho$-a.e. $\left(\int_{k}(1-\alpha) d|\rho|=0\right.$ and $1-\alpha \geq 0$ forces $1-\alpha \equiv 0|\rho|$-a.e.; see Rudin $\left.[15,1.39(\mathrm{a})]\right)$. Thus $\beta \equiv 0 \rho$-a.e. So $\rho=|\rho|$.

\section{Semihypergroups}

Here a usable notion of semihypergroup is defined and some basic theory is developed.

DEFINITION. A semihypergroup $K$ is a locally compact Hausdorff space with the following properties.

(a) The space $M(K)$ of finite complex regular Borel measures has a product * making it a Banach algebra.

(b) The product of probability measures on $K$ is always a probability measure.

(c) The product is weak-* jointly continuous on the unit ball of $M(K)$.

Note that a compact semihypergroup is also a semiconvo as defined by Jewett $\left[9\right.$, Section 3]. For a semihypergroup $K$ define $K^{\wedge} \subseteq C(K)$ to be $\{f \in$ $C(K): \int_{K} f d p_{k} * p_{l}=f(k) f(l)$ for all $\left.k, l \in K\right\}$. We say $K$ is unital if there exists $e \in K$ such that $p_{e}$ is a unit for the Banach algebra $M(K)$.

LEMMA 2.1. Let $K$ be a locally compact Hausdorff space. If $L: M(K) \rightarrow \mathbb{C}$ is weak-* continuous and linear, then there is a function $h \in C_{0}(K)$ such that $L(\nu)=\int_{K} h d \nu$ for all $\nu \in M(K)$.

ProOF. This follows from the identification of $C(K)^{*}$ with $M(K)$. 
PROPOSITION 2.2. If $K$ is a semihypergroup, we may write

$$
\int_{K} \int_{K} \int_{K} f d p_{k} * p_{l} d \mu(k) d \nu(l)=\int_{K} f d \mu * \nu
$$

for all $f \in C_{0}(K), \mu, \nu \in M(K)$.

Proof. Let $f \in C_{0}(K)$ and $\mu, \nu \in M(K)$. Consider the map $\eta \rightarrow \int_{K} f d \mu * \eta$. Since $\eta \rightarrow \mu * \eta$ is weak-* continuous (which follows from assumption (c) on $K$ ), the map is linear and weak-* continuous on $M(K)$. So by the lemma, there exists $h \in C_{0}(K)$ such that $\eta \rightarrow \int_{K} f d \mu * \eta$ is the map $\eta \rightarrow \int_{K} h d \eta$. That is, $\int_{K} f d \mu * \nu=\int_{K} h d \nu$. Now $h(l)=\int_{K} h d p=\int_{K} f d \mu * p_{l}$. So $\int_{K} f d \mu * \nu=$ $\int_{K}\left(\int_{K} f d \mu * p_{l}\right) d \nu(l)$.

Now consider the map $\eta \rightarrow \int_{K} f d \eta * p_{l}$. As before, there exists $g \in C_{0}(K)$ such that $\int_{K} f d \eta * \mu=\int_{K} g d \eta$. Now $g(k)=\int_{K} g d p_{k}=\int_{K} f d p_{k} * p_{l}$. So $\int_{K} f d \mu * p_{l}=\int_{K} \int_{K} \int_{K} f d p_{k} * p_{l} d \mu(k) d \nu(l)$.

Note. As with hypergroups, we often write $f(k * l)=\int_{K} f d p_{k} * p_{l}$ for $k, l \in K$ and $f \in C_{0}(K)$, even though $k * l$ does not represent a point in $K$. By assumption (c) for semihypergroups, we know that a $f(k * l)$ is jointly continuous on $K \times K$. With this notation, the conclusion of the proposition reads

$$
\int_{K} \int_{K} f(k * l) d \mu(k) d \nu(l)=\int_{K} f d \mu * \nu
$$

Also $f \in K^{\wedge}$ means $f(k * l)=f(k) f(l)$ for all $k, l \in K$.

Warning: $K^{\wedge}$ is not contained in $C_{0}(K)$, unless $K$ is compact.

DEFINITION. Suppose $H$ and $K$ are semihypergroups. Then a continuous map $\phi: H \rightarrow K$ is a semihypergroup homomorphism if for all $f \in C_{0}(K)$ and all $k, l \in H$, then $f \circ \phi \in C_{0}(H)$ and $(f \circ \phi)(k * l)=f(\phi(k) * \phi(l))$.

REMARK. If $H$ and $K$ are semigroups, this becomes $f(\phi(k l))=f(\phi(k) \phi(l))$ (so $\phi(k l)=\phi(k) \phi(l)$ ). Since $k * l$ has no independent meaning, we must formulate this definition using functions.

Note that $\phi$ extends in a natural way to be an algebra homomorphism of measure algebras.

Proposition 2.3. The map $\mu \rightarrow \mu \circ \phi^{-1}: M(H) \rightarrow M(K)$ is a normcontinuous and weak-* continuous algebra and $L$-homomorphism, which sends point-masses to point-masses.

ProOF. Clearly, point-masses are sent to point-masses, and the map is a norm-continuous $L$-homomorphism by Taylor [17, 2.2.2]. Easily the map is weak* continuous. To show that $\mu \rightarrow \mu \circ \phi^{-1}$ is an algebra homomorphism, select 
$\mu, \nu \in M(H)$ and $f \in C_{0}(K)$. Then

$$
\begin{aligned}
\int_{K} f d(\mu * \nu) \circ \phi^{-1} & =\int_{K} \int_{K}(f \circ \phi)(k * l) d \mu(k) d \nu(l) \\
& =\int_{K} \int_{K} f(\phi(k) * \phi(l)) d \mu(k) d \nu(l) \\
& =\int_{K} \int_{K} f(k * l) d \mu \circ \phi^{-1}(k) d \nu \circ \phi^{-1}(l) \\
& =\int_{K} f d\left(\mu \circ \phi^{-1}\right) *\left(\nu \circ \phi^{-1}\right)
\end{aligned}
$$

\section{The structure-strong condition for a hypergroup measure algebra}

The following condition enables us to build a close analogue of Taylor's theorem $[17,3.2 .3]$ for hypergroup measure algebras.

DEFINITION. A commutative hypergroup measure algebra $M(H)$ is structurestrong if the norm-closure of the linear span of $\Delta M(H)$ in $M(H)^{*}$ is a commutative unital $B^{*}$-algebra.

Note. This condition is in force for commutative convolution measure algebras (see Taylor [17, 3.2.2]). If a hypergroup is not a semigroup, its measure algebra will not be a commutative convolution measure algebra. This is readily shown by checking the definition of Taylor $[17,3.1 .2]$. (Use $[17,2.5 .2]$.)

EXAMPLES. Some examples of hypergroups whose measure algebras satisfy the structure-strong condition are given by countable discrete hypergroups whose hypergroup characters form a hypergroup; see Jewett $[9,7.3]$. For them, the hypergroup algebra $L^{1}(H)$ and the measure algebra coincide, and the spectrum is just the set of hypergroup characters. (See Jewett $[9,6.3]$.) A discrete countable commutative hypergroup which fails to satisfy this condition appears in Lasser [10, Remark 1 following Proposition 1]. Lasser defines the hypergroup analogue of almost periodic functions. His example is of a hypergroup whose almost periodic functions do not span an algebra (under pointwise product). In fact, Lasser shows this by showing that the product of two certain hypergroup characters is not almost periodic, but all hypergroup characters are almost periodic (as is any linear combination).

Examples which are less trivial in the sense that their measure algebras differ from their hypergroup (function) algebras are developed in Sections 4 and 5 of this paper. 


\section{Structure semihypergroups}

We are now ready to state and prove the following theorem. The proof follows Rennison's proof of Taylor's theorem [17, 3.2.3] (see Rennison [13]).

THEOREM 4.1. Suppose $H$ is a (locally compact Hausdorff) commutative hypergroup such that $M(H)$ is structure-strong. Then there is a compact commutative semihypergroup $K$, known as the structure semihypergroup of $M(H)$, and an order-preserving linear algebra homomorphism $\mu \rightarrow \mu_{K}: M(H) \rightarrow M(K)$ such that

(a) Every $f \in \Delta M(H)$ arises as $f(\mu)=\int_{K} \hat{f} d \mu_{K}$ for all $\mu \in M(H)$, for some $\hat{f} \in K^{\wedge}$, and $\Delta M(H)$ is exactly $K^{\wedge}$ in this way,

(b) The space $K^{\wedge}$ separates points of $K$,

(c) The image of $M(H)$ in $M(K)$ is weak-* dense.

PROOF. The assumption that $M(H)$ is structure-strong is the assumption that $A=$ closed linear span of $\Delta M(H)$ in $M(H)^{*}$ is a commutative unital $B^{*}$ algebra. Let $K=\Delta A$. The space $K$ will be the structure semihypergroup. By the Gelfand-Naimark theorem, $A=C(\Delta A)=C(K)$. By the Riesz representation theorem, $A^{*}=M(K)$. Let $A^{0} \subseteq M(H)^{* *}$ be the annihilator of $A \subseteq M(H)^{*}$. We proceed with the help of five lemmas.

LEMMA 4.1.1. If $f \in \Delta M(H)$ and $\mu \in M(H)$ then

(1) $\langle f, \mu\rangle=f(\mu) f$.

If $f \in \Delta M(H)$ and $F \in M(H)^{* *}$ then

(2) $[F, f]=F(f) f$.

If $f \in M(H)^{*}$ and $\mu \in M(H)$, then

(3) $\|\langle f, \mu\rangle\| \leq\|f\|\|\mu\|$.

If $f \in M(H)^{*}$ and $F \in M(H)^{* *}$, then

(4) $\|[F, f]\| \leq\|F\|\|f\|$.

Proof. (1) We have $\langle f, \mu\rangle(\nu)=f(\mu * \nu)=f(\mu) f(\nu)$ for all $\nu \in M(H)$. Thus $\langle f, \mu\rangle=f(\mu) f$. (2) We have $[F, f](\mu)=F(\langle f, \mu\rangle)=F(f(\mu) f)=f(\mu) F(f)$. Thus $[F, f]=F(f) f$, using (1).

(3), (4) These follow from the definition of the Arens product. This completes Lemma 1.

Lemma 4.1.2. If $f \in A$ and $\mu \in M(H)$ then $\langle f, \mu\rangle \in A$.

Proof. First consider $f \in \Delta M(H)$. Then by $(1),\langle f, \mu\rangle=f(\mu) f \in A$. 
For $\alpha_{1}, \ldots, \alpha_{n} \in \mathbb{C}$ and $g_{1}, \ldots, g_{n} \in \Delta M(H)$, if $f=\sum_{1}^{n} \alpha_{i} g_{i}$ then using (1), $\langle f, \mu\rangle=\sum_{1}^{n} \alpha_{i} g_{i}(\mu) g_{i} \in A$.

Now if $f \in A$ is arbitrary, for $\varepsilon>0$ there is $f^{\prime} \in A$ which is a finite linear combination of elements of $\Delta M(H)$, such that $\left\|f-f^{\prime}\right\|<\varepsilon$. Then $\left\|\langle f, \mu\rangle-\left\langle f^{\prime}, \mu\right\rangle\right\|=\left\|\left\langle f-f^{\prime}, \mu\right\rangle\right\| \leq\left\|f-f^{\prime}\right\|\|\mu\| \leq \varepsilon\|\mu\|$ using (3). This means $\langle f, \mu\rangle$ is arbitrarily close in norm to members of $A$. So $\langle f, \mu\rangle \in A$.

LEMMA 4.1.3. If $f \in A$ and $F \in M(H)^{* *}$ then $\langle F, f\rangle \in A$.

PROOF. Similar to Lemma 4.1.2; use (4) of Lemma 4.1.1.

LEMMA 4.1.4. The space $A^{0}$ is a closed ideal in $M(H)^{* *}$. Also $A^{*}$ and $M(H)^{* *} / A^{0}$ are isometrically isomorphic Banach spaces, when $M(H)^{* *} / A^{0}$ is given the canonical quotient norm. And $M(H)^{* *} / A^{0}$ is commutative.

Proof. First $A^{0}$ is an ideal: Let $F \in A^{0}, G \in M(H)^{* *}$. Let $f \in A$. We need to show $F G(f)=0$. Now $F G(f)=F([G, f])=0$ since by Lemma 4.1.3, $[G, f] \in A$. Also, $G F(f)=F([F, f])=G(0)=0$ since $[F, f](\mu)=F(\langle f, \mu\rangle)=0$ for all $\mu \in M(H)$ since by Lemma 4.1.2, $\langle f, \mu\rangle \in A$. Thus $A^{0}$ is a (two-sided) ideal.

Now $A^{0}$ is closed: suppose $F$ belongs to the norm-closure of $A^{0}$. Then for $\varepsilon>0$ there exists $G \in A^{0}$ such that $\|F-G\|<\varepsilon$. Select $f \in A$. Then $|F(f)-G(f)|<\varepsilon|| f \|$, or $|F(f)|<\|f\| \varepsilon$. Then $\varepsilon$ arbitrary forces $F(f)=0$. Thus $F \in\left(A^{0}\right)^{-}$. So $A^{0}$ is closed.

Then $M(H)^{* *} / A^{0}$ is a Banach algebra under the canonical norm:

$$
\left\|f+A^{0}\right\|=\inf \left\{\|f+g\|: g \in A^{0}\right\} .
$$

(This depends merely upon $A^{0}$ being a two-sided ideal of $M(H)^{* *}$.) Using the Hahn-Banach theorem, it may be shown for $F \in M(H)^{* *}$ that $\left\|\left.F\right|_{A}\right\|=$ $\left\|F+A^{0}\right\|$. So $A^{*}$ and $M(H)^{* *} / A^{0}$ are isometrically isomorphic Banach algebras.

Now it remains to show that $M(H)^{* *} / A^{0}$ is commutative. Let $F, G \in M(H)^{* *}$. It is enough to show that $F G-G F \in A^{0}$. Now for $f \in \Delta M(H)$ we have $F G(f)=$ $F([G, f])=F(G(f) f)=G(f) F(f)$ (using Lemma 1) so $(F G-G F)(f)=0$. Since $F G-G F$ is bounded and linear, this must hold for any $f \in A$. Thus $F G-G F \in A^{0}$. So $F G=G F$ in $M(H)^{* *} / A^{0}$. Thus that algebra is commutative, and Lemma 4.1.4 is complete.

Thus $A^{*}$ inherits the commutative Banach algebra structure of $M(H)^{* *} / A^{0}$. We may use the Arens product to write down the product of $A^{*}$; this is well defined by the lemmas above. 
Now define the map $\mu \rightarrow \mu_{K}: M(H) \rightarrow M(K)$, as follows:

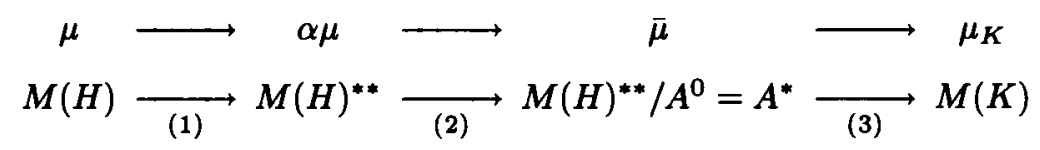

where

(1) is canonical

(2) is canonical: $\bar{\mu}$ is $\alpha \mu$ restricted to $A$

(3) is the adjoint of the inverse Gelfand transform followed by the Riesz representation theorem.

Now by Taylor $[17,2.4 .3]$, this map $\mu \rightarrow \mu_{K}$ is order-preserving and the image is weak-* dense in $M(K)$. (Set $A=$ closed linear span of $\Delta M(H)$, and $Y=K=\Delta A$.) This establishes (c) of this theorem.

We also have the equation: For $f \in A, \mu \in M(H), f(\mu)=\int_{K} \hat{f} d \mu_{K}(\hat{f} \in$ $C(K)$ being the Gelfand transform of $f$ ):

$$
\begin{aligned}
\int_{K} \hat{f} d \mu_{K} & =\mu_{K}(\hat{f}) \quad \text { (Riesz representation theorem) } \\
& =\alpha \mu(f) \quad \text { (definition of map (3)) } \\
& =f(\mu) \quad \text { (definition of maps (1) and (2)). }
\end{aligned}
$$

Now we show that the product of two probability measures is a probability measure: Let $\mu, \nu \in M(K)$ be probability measures. Let $F, G \in A^{*}$ correspond to $\mu, \nu$ respectively. $F(1)=G(1)=1$ since $\int_{K} 1 d \mu=\int_{K} 1 d \nu=1$. (Note $1 \in M(H)^{*}$ is the unit of that algebra and has Gelfand transform in $C(K)$ equal to the constant one function.) By Lemma 4.1.1, since 1 is a multiplicative functional, $[G, 1]=1$. So $F G(1)=F([G, 1])=F(1)=1$. This means $\int_{K} 1 d \mu * \nu=1$. Then $\|\mu * \nu\| \leq\|\mu\|\|\nu\|=1$ forces $\mu * \nu$ to be a probability measure by Lemma 1.1 .

LEMMA 4.1.5. For $\mu, \nu \in M(K), f \in C(K)$,

$$
\int_{K} f d \mu * \nu=\int_{K} \int_{K}\left(\int_{K} f d p_{k} * p_{l}\right) d \mu(k) d \nu(l) .
$$

Proof. Let $F, G \in A^{*}$ correspond to $\mu, \nu$ respectively. Then $\int_{K} f d \mu * \nu=$ $F G(f)$ where we write $f \in A$ for the inverse Gelfand transform of $f \in C(K)$. Also $\int_{K} f d p_{k} * p_{l}=k l(f)\left(k l \in A^{*}\right.$ since $\left.k, l \in K=\Delta A=A^{*}\right)$. Write as usual $f(k * l)=\int_{K} f d p_{k} * p_{l}$. Then the map

$$
k \rightarrow f(k * l)
$$

is the map

$$
k \rightarrow k l(f)=k([l, f])
$$


or

$$
k \rightarrow[l, f]^{\wedge}(k)
$$

so this is the map whose inverse Gelfand transform is $[l, f]$. Now $l \in A^{*}$ and $f \in A$ so by Lemma 4.1.5, $[l, f] \in A$. Then $[l, f]^{\wedge} \in C(K)$. So $k \rightarrow f(k * l)$ is a continuous function on $K$. So the integral $\int_{K} f(k * l) d \mu(k)$ is well defined. Since $k \rightarrow f(k * l)$ is $[l, f]^{\wedge}$ in $C(K)$, and $F \in A^{*}$ corresponds to $\mu$, this integral is $F([l, f])=F l(f)=l F(f)\left(A^{*}\right.$ is commutative $)=l([F, f])$. Then the map $l \rightarrow$ $\int_{K} f(k * l) d \mu(k)$ is $l \rightarrow l([F, f])$ or $l \rightarrow[F, f]^{\wedge}(l)$. Since by Lemma 4.1.4, $[F, f] \in$ $A$, this map is continuous. So the integral $\int_{K} \int_{K}\left(\int_{K} f d p_{k} * p_{l}\right) d \mu(k) d \nu(l)$ is well defined. So we may now compute $\int_{K} \int_{K}\left(\int_{K} f d p_{k} * p_{l}\right) d \mu(k) d \nu(l)=F([F, f])=$ $G F(f)=F G(f)=\int_{K} f d \mu * \nu$. This completes Lemma 4.1.5.

With Lemma 4.1.5,we may show that if $f \in K^{\wedge}$ then the inverse Gelfand transform $f \in \Delta M(H) \subseteq A$. (Recall $K^{\wedge}=\{f \in C(K): f(k * l)=f(k) f(l)$ for all $k, l \in K\}$.) For $\mu, \nu \in M(H)$ we have

$$
\begin{aligned}
f(\mu * \nu) & =\int_{K} f d \mu_{K} * \nu_{K}=\int_{K} \int_{K} f(k * l) d \mu_{K}(k) d \nu_{K}(l) \\
& =\int_{K} \int_{K} f(k) f(l) d \mu_{K}(k) d \nu_{K}(l) \\
& =\left(\int_{K} f(k) d \mu_{K}(k)\right)\left(\int_{K} f(l) d \mu_{K}(l)\right) \\
& =f(\mu) f(\nu)
\end{aligned}
$$

where we use the multiplicativity of $\mu \rightarrow \mu_{K}$ in the second equality, and Lemma 5 in the third.

Now we wish to show that $f \in K$ for $f \in \Delta M(H)$. Consider the linear functional $\mu \rightarrow \int_{K} f d \mu$ defined on $M(K)$. It is multiplicative. To see this, proceed as in Civin and Yood $[1,3.6]$ : The map is the same as $F \rightarrow F(f)$ for $F \in A^{*}$. For $G, F \in A^{*}, G F(f)=G([F, f])=G(F(f) f)=F(f) G(f)=$ $G(f) F(f)$ using Lemma 4.1.1 (2). Formulated for $M(K)$, we have $\int_{K} f d \mu * \nu=$ $\left(\int_{K} f d \mu\right)\left(\int_{K} f d \nu\right)$ for all $\mu, \nu \in M(K)$. By Lemma 5 this is

$$
\begin{aligned}
\int_{K} \int_{K} f(k * l) d \mu(k) d \nu(l) & =\left(\int_{K} f(k) d \mu(k)\right)\left(\int_{K} f(l) d \nu(l)\right) \\
& =\int_{K} \int_{K} f(k) f(l) d \mu(k) d \nu(l)
\end{aligned}
$$

Since this holds for any $\mu, \nu \in M(K)$, we conclude $f(k * l)=f(k) f(l)$ for all $k, l \in K$. Thus $f \in \Delta M(H) \Rightarrow f \in K$. This establishes (a). Since $\Delta M(H)$ generates $A, K^{\wedge}$ generates $C(K)$. This establishes (b).

All that remains is to verify that multiplication on the unit ball of $M(K)$ is weak-* jointly continuous. 
Let $U(\varepsilon, f, \mu)=\left\{\nu \in A_{1}^{*}:|\nu(f)-\mu(f)|<\varepsilon\right\}$, for $\varepsilon>0, f \in A, \mu \in A_{1}^{*}$. Then of course $\left\{U(\varepsilon, f, \mu): \varepsilon>0, f \in A, \mu \in A_{1}^{*}\right\}$ is a subbasis for $A_{1}^{*}$ in the weak-* topology. Now $\left\{U(\varepsilon, f, \mu): \varepsilon>0, f \in \Delta M(H), \mu \in A_{1}^{*}\right\}$ is a subbasis for $A_{1}^{*}$ as well.

So it suffices to check continuity against subbasis members of form $U(\varepsilon, f, \mu)$ where $f \in \Delta M(H)$. Consider $\mu, \nu \in A_{1}^{*}$ and a subbasis neighborhood of $\mu * \nu$ given by $U(\varepsilon, f, \mu * \nu)$ where $f \in \Delta M(H)$ and $\varepsilon>0$. Then $U(\varepsilon / 2, f, \mu) *$ $U(\varepsilon / 2, f, \nu) \subseteq U(\varepsilon, f, \mu * \nu)$. This is true since if $\left|\mu^{\prime}(f)-\mu(f)\right|<\varepsilon / 2$ and $\left|\nu^{\prime}(f)-\nu(f)\right|<\varepsilon / 2$, then

$$
\begin{aligned}
\mid \mu^{\prime} * \mu(f)-\nu^{\prime} & * \nu(f)|=| \mu^{\prime}(f) \mu(f)-\nu^{\prime}(f) \nu(f) \mid \\
& =\left|\left(\mu^{\prime}(f)-\mu(f)\right) \nu^{\prime}(f)+\left(\nu^{\prime}(f)-\nu(f)\right) \mu(f)\right| \\
& \leq\left|\mu^{\prime}(f)-\mu(f)\right|+\left|\nu^{\prime}(f)-\nu(f)\right|<\varepsilon \quad \text { using Lemma 4.1.1 (2). }
\end{aligned}
$$

Thus the product of $A_{1}^{*}=M(K)_{1}$ is weak-* jointly continuous. So $K$ is a semihypergroup (and commutative since $M(K)=A^{*}$ is a commutative algebra). So the theorem is done.

The following theorem shows that in a natural way, structure semihypergroup homomorphisms are unique.

THEOREM 4.2. Suppose $H$ and $J$ are structure-strong hypergroups with respective structure semihypergroups $K$ and $Y$. Suppose $\phi: M(H) \rightarrow M(J)$ is an algebra and L-homomorphism. Then there exists $\alpha: K \rightarrow Y$, a semihypergroup homomorphism, such that the following diagram commutes

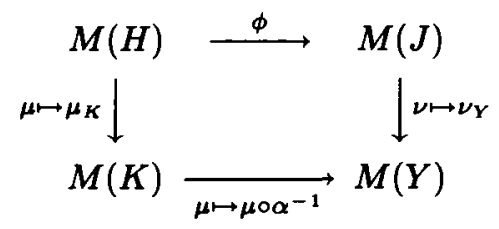

Proof. Since $\phi: M(H) \rightarrow M(J)$ is an algebra homomorphism, the adjoint map $\phi^{*}: M(J)^{*} \rightarrow M(H)^{*}$ maps $\{0\} \cup \Delta M(J)$ into $\{0\} \cup \Delta M(H)$. (For $\mu, \nu \in M(H), f \in \Delta M(J), \phi^{*}(f)(\mu * \nu)=f(\phi(\mu * \nu))=f(\phi(\mu) * \phi(\nu))=$ $f(\phi(\mu)) f(\phi(\nu))=\phi^{*}(f)(\mu) \phi^{*}(f)(\nu)$ so $\phi^{*}(f) \in \Delta M(H) \cup\{0\}$.)

Then there is a unital algebra homomorphism $\psi: C(Y) \rightarrow C(K)$ (just $\phi^{*}$ with $A=$ closed linear span of $\Delta M(H)$ and $B=$ closed linear span of $\Delta M(J)$ identified with $C(K), C(Y)$ respectively). Then there is a continuous map $\alpha: K \rightarrow Y$ such that $(\psi f)(k)=f(\alpha(k))$ for all $f \in C(Y), k \in K$. (This is true for any unital algebra homomorphism $\phi: C(X) \rightarrow C(Y)$ where $X$ and $Y$ are arbitrary locally compact Hausdorff spaces.)

This map makes the diagram commute: Pick $\mu \in M(H)$. Consider any $f \in$ $C(Y)$. Then $\int_{Y} f d(\phi \mu)_{Y}=f(\phi \mu)=\phi^{*}(f)(\mu)=\int_{K} \psi(f) d \mu_{K}=\int_{K} f \circ \alpha d \mu_{K}=$ 
$\int_{K} f d \mu_{K} \circ \alpha^{-1}$ (note we write $f$ for the inverse Gelfand transform of $f$ ). Thus $(\phi \mu)_{Y}=\mu_{K} \circ \alpha^{-1}$.

To see that $\alpha$ is a hypergroup homomorphism, note that $f \rightarrow f \circ \alpha: C(Y) \rightarrow$ $C(K)$ maps $Y^{\wedge}$ to $K^{\wedge}$ (since $\phi^{*}: B \rightarrow A$ takes $\Delta M(J) \cup\{0\}$ to $\Delta M(H) \cup\{0\}$ ). Then for $f \in Y^{\wedge},(f \circ \alpha)(k * l)=(f \circ \alpha)(k)(f \circ \alpha)(l)=f(\alpha(k) * \alpha(l))$ for all $k, l \in K$. Since $C(Y)$ is the closed linear span of $Y^{\wedge}$, it is easy to see that this is true for all $f \in C(Y)$.

\section{The hypergroup of $B$-orbits of $G$, where $B$ is a finite solvable group and $G$ is a locally compact abelian group}

These interesting examples of hypergroups resemble group measure algebras in that the product of any two point-masses has finite support. These measure algebras will be shown to satisfy the structure-strong condition.

First we consider how $B$ acts on various algebras. We will customarily consider $B=\left\{e, \beta_{1}, \ldots, \beta_{n-1}\right\}$ as a solvable group of order $n$, acting on $G$ as automorphisms. We may also think of $B$ as acting on $C_{0}(G)$, by $(f \circ \beta)(x)=f(\beta(x))$ for all $f \in C_{0}(G), \beta \in B, x \in G$. So if $f \in C_{0}(G)$, then $f \circ \beta \in C_{0}(G)$. We say $f \in C_{0}(G)$ is $B$-invariant if $f \circ \beta=f$ for all $\beta \in B$. The group $B$ acts on $M(G)$ by $(\mu \circ \beta)(E)=\mu(\beta(E))$ for $\mu \in M(G), \beta \in B$, and Borel $E \subseteq G$. If instead $M(G)$ is thought of as $C_{0}(G)^{*}, \mu \circ \beta$ is the linear functional defined by $(\mu \circ \beta)(f)=\mu\left(f \circ \beta^{-1}\right)$ for $\mu \in C_{0}(G)^{*}, f \in C_{0}(G), \beta \in B$. (These agree since $\int_{G} f d \mu \circ \beta=\int_{G} f \circ \beta^{-1} d \mu$ for all $\beta \in B, f \in C_{0}(G), \mu \in M(G)$.) For $\mu \in M(G)$, we say $\mu$ is $B$-invariant if $\mu \circ \beta=\mu$ for all $\beta \in B$. The group $B$ acts on $M(G)^{*}$ in a similar fashion: $(f \circ \beta)(\mu)=f(\mu \circ \beta)$ for all $f \in M(G)^{*}$, $\mu \in M(G), \beta \in B$. We say $f \in M(G)^{*}$ is $B$-invariant if $f=f \circ \beta$ for all $\beta \in B$.

The following lemma will often be used without explicit reference:

LEMMA 5.1. If $\mu, \nu \in M(G),(\mu * \nu) \circ \beta=(\mu \circ \beta) *(\nu \circ \beta)$ for all $\beta \in B$.

Proof. Consider $f \in C_{0}(G)$. Then for $\beta \in B$,

$$
\begin{array}{rl}
\int_{G} f & d(\mu * \nu) \circ \beta=\int_{G} f \circ \beta^{-1} d \mu * \nu \\
& =\int_{G} \int_{G} f\left(\beta^{-1}(x+y)\right) d \mu(x) d \nu(y) \int_{G} \int_{G} f\left(\beta^{-1}(x)+\beta^{-1}(y)\right) d \mu(x) d \nu(y) \\
& =\int_{G} \int_{G} f(x+y) d \mu(\beta(x)) d \nu(\beta(y)) \\
& =\int_{G} f d(\mu \circ \beta) *(\nu \circ \beta) .
\end{array}
$$

Thus $(\mu * \nu) \circ \beta=(\mu \circ \beta) *(\nu \circ \beta)$. 
A corollary of this result is that if $\mu, \nu \in M(G)$ are $B$-invariant, so is $\mu * \nu$. Another corollary is that if $f \in \Delta M(G)$, then $f \circ \beta \in \Delta M(G)$ for any $\beta \in B$. Of course it is clear that in $C_{0}(G),(f \circ \beta)(g \circ \beta)=(f g) \circ \beta$ for all $\beta \in B$ (pointwise product). Also, we have the following lemma.

Lemma 5.2. Suppose $f, g \in M(G)^{*}$. Then $(f g) \circ \beta=(f \circ \beta)(g \circ \beta)$ for any $\beta \in B$ (where the product is the Sreider product of generalized functions, described above in Section 1).

Proof. First, for any $f \in M(G)^{*}$ consider its generalized function $\left\{f_{\mu}\right\}$. Then for $\mu \in M(G), \nu \ll \mu,(f \circ \beta)(\nu)=\int_{G}(f \circ \beta)_{\mu} d \nu$ and $(f \circ \beta)(\nu)=$ $f(\nu \circ \beta)=\int_{G} f_{\mu \circ \beta} d \nu \circ \beta=\int_{G} f_{\mu \circ \beta} \circ \beta^{-1} d \nu$ (easily $\nu \circ \beta \ll \mu \circ \beta$ ). Therefore $(f \circ \beta)_{\mu}=f_{\mu \circ \beta} \circ \beta^{-1} \mu$-a.e.. Then for $\beta \in B, \mu \in M(G)$, we have by the definition of the product of generalized functions $((f g) \circ \beta)_{\mu}=\left((f g)_{\mu \circ \beta}\right) \circ \beta^{-1}=$ $\left(f_{\mu \circ \beta} g_{\mu \circ \beta}\right) \circ \beta^{-1}=\left(f_{\mu \circ \beta} \circ \beta^{-1}\right)\left(g_{\mu \circ \beta} \circ \beta^{-1}\right)\left(f_{\mu \circ \beta}\right.$ and $g_{\mu \circ \beta}$ are just $L^{\infty}(\mu \circ \beta)$ functions, so composition by $\beta^{-1}$ behaves as usual for pointwise products of functions). Thus $((f g) \circ \beta)_{\mu}=(f \circ \beta)_{\mu}(g \circ \beta)_{\mu}=[(f \circ \beta)(g \circ \beta)]_{\mu}$. (These inequalities are $\mu$-a.e.) Thus $(f g) \circ \beta$ and $(f \circ \beta)(g \circ \beta)$ have the same generalized functions, so are equal.

We are interested in studying $\Delta M\left(G_{B}\right)$, the spectrum of $M\left(G_{B}\right)$. The algebra $M\left(G_{B}\right)$ is identified with $M(G)_{B}$, the closed subalgebra of $B$-invariant measures in $M(G)$. Then it is natural to identify $\Delta M\left(G_{B}\right)$ with $\Delta\left(M(G)_{B}\right)=$ $\left\{f \in M(G)^{*}: f\right.$ is $B$-invariant, and multiplicative on products of $B$-invariant measures\}. (Just extend $f$, defined and multiplicative on $M(G)_{B}$, to be a member $g$ of $\Delta\left(M(G)_{B}\right)$, by $g(\mu)=f\left(\mu_{B}\right)$ for all $\mu \in M(G)$, where $\mu_{B}=$ $1 / n \sum_{\beta \in B} \mu \circ \beta$ is easily checked to be $B$-invariant.) For $f \in M(G)^{*}, \nu \in M(G)$, let $f_{\nu} \in M(G)^{*}$ be defined by $f_{\nu}(\mu)=f(\nu * \mu)$ for all $\mu \in M(G)$. (This is actually the same as $\langle f, \nu\rangle$ in the Arens product.)

We are now ready for the following theorem.

THEOREM 5.3. Suppose $A$ and $B$ are finite groups of automorphisms on a locally compact abelian group $G$, with $A$ a normal subgroup of $B$. Suppose $B / A=\mathbf{Z}_{p}$ for $p$ a prime number. Suppose $f \in M(G)^{*}$ is $B$-invariant and multiplicative on $B$-invariant measures. Then there exists $\eta \in M(G)$ such that $f_{\eta}$ is multiplicative on $A$-invariant measures and is $A$-invariant.

Proof. First it makes sense to think of $B / A$ as acting on $A$-invariant measures, by $\mu \circ(\beta A)=\mu \circ \beta(\mu A$-invariant, $\beta \in B)$. This is well defined because $\mu \circ\left(\beta \alpha_{1}\right)=\mu \circ\left(\beta \alpha_{2}\right)$ for all $\alpha_{1}, \alpha_{2} \in A$. (This follows from the normality of $A$ in $B$.) 
Now $B / A=Z_{p}$, so there exists $\beta \in B$ such that $\beta A$ generates $B / A$ as a cyclic group of order $p$. From now on, when we write $\beta$ or $\beta A$, we refer to that generator.

Define $\mu \in M(G)$ to be of type $k$ if $\mu$ is $A$-invariant and $\mu \circ(\beta A)=\mu \circ \beta=$ $e^{2 \pi i k / p} \mu$. Note $\mu$ is of type 0 means $\mu$ is $B$-invariant. So $f$ is multiplicative on products of type 0 measures.

Suppose $\mu$ is any $A$-invariant measure. Then

CLAIM 1. We have $\mu=\mu_{0}+\mu_{1}+\cdots+\mu_{p-1}$ where $\mu_{k}$ is of type $k$ ( $k=$ $0,1, \ldots, p-1)$. This decomposition is unique and in fact

$$
\mu_{k}=\frac{1}{p} \sum_{l=0}^{p-1} e^{-2 \pi i l k / p} \mu \circ\left(\beta^{l} A\right) \quad(k=0,1, \ldots, p-1) .
$$

PROOF. We need $\mu_{k}$ of type $k$ for $k=0,1, \ldots, p-1$ so that $\mu=\mu_{0}+\mu_{1}+$ $\cdots+\mu_{p-1}$. Consider the system of linear equations, for Borel $E \subseteq G$,

$$
\begin{aligned}
\mu(E)= & \mu_{0}(E)+\mu_{1}(E)+\mu_{2}(E)+\cdots+\mu_{p-1}(E) \\
\mu \circ \beta(E)= & \mu_{0} \circ \beta(E)+\mu_{1} \circ \beta(E)+\mu_{2} \circ \beta(E)+\cdots+\mu_{p-1} \circ \beta(E) \\
\mu \circ \beta^{2}(E)= & \mu_{0} \circ \beta^{2}(E)+\mu_{1} \circ \beta^{2}(E)+\mu_{2} \circ \beta^{2}(E)+\cdots+\mu_{p-1} \circ \beta^{2}(E) \\
\mu \circ \beta^{-1}(E)= & \mu_{0} \circ \beta^{p-1}(E)+\mu_{1} \circ \beta^{p-1}(E)+\mu_{2} \circ \beta^{p-1}(E) \\
& +\cdots+\mu_{p-1} \circ \beta^{p-1}(E)
\end{aligned}
$$

which becomes (where $\lambda=e^{2 \pi i / p}$ ),

$$
\begin{aligned}
\mu(E) & =\mu_{0}(E)+\mu_{1}(E)+\mu_{2}(E)+\cdots+\mu_{p-1}(E) \\
\mu \circ \beta(E)= & \mu_{0}(E)+\lambda \mu_{1}(E)+\lambda^{2} \mu_{2}(E)+\cdots+\lambda^{p-1} \mu_{p-1}(E) \\
\mu \circ \beta^{2}(E)= & \mu_{0}(E)+\lambda^{2} \mu_{1}(E)+\lambda^{4} \mu_{2}(E)+\cdots+\lambda^{2(p-1)} \mu_{p-1}(E) \\
& \cdots \\
\mu \circ \beta^{p-1}(E)= & \mu_{0}(E)+\lambda^{p-1} \mu_{1}(E)+\lambda^{2(p-1)} \mu_{2}(E)+\cdots+\lambda^{(p-1)(p-1)} \mu_{p-1}(E)
\end{aligned}
$$

Now this system can be solved uniquely for the $\mu_{k}(E)$ in terms of the $\mu \circ \beta^{l}(E)$, provided

$$
\operatorname{det}\left[\begin{array}{ccccc}
1 & 1 & 1 & \cdots & 1 \\
1 & \lambda & \lambda^{2} & \cdots & \lambda^{p-1} \\
1 & \lambda^{2} & \lambda^{2} & \cdots & \lambda^{2(p-1)} \\
& \cdots & & & \\
1 & \lambda^{p-1} & \lambda^{2(p-1)} & \cdots & \lambda^{(p-1)(p-1)}
\end{array}\right] \neq 0 .
$$

In fact, $\operatorname{det} \neq 0$ since the columns form an orthogonal basis for $\mathbb{C}^{n}$. (The columns are actually the group characters for $\mathbf{Z}_{p}$.) This forces the $\mu_{k}$ to be unique. To obtain the claimed formula for $\mu_{k}$, fix measurable $E \subseteq G$ and define $f \in C\left(\mathbf{Z}_{p}\right)$ (for $\mu$ and $E$ ) by $f(k)=\mu\left(\beta^{k}(E)\right.$ ). Then $f(k)=\sum_{n=0}^{p-1} \hat{f}(n) e^{2 \pi i k n / p}$ 
where $\hat{f}(n)=1 / p \sum_{j=0}^{p-1} f(j) e^{-2 \pi i n j / p}=1 / p \sum_{j=0}^{p-1} \mu\left(\beta^{j}(E)\right) e^{-2 \pi i n j / p}$. (This is just the inversion theorem for the Fourier transform on $C\left(\mathbf{Z}_{p}\right)$.) Now let $k=0$. We get $\mu(E)=f(0)=\sum_{n=0}^{p-1}\left(1 / p \sum_{j=0}^{p-1} \mu\left(\beta^{j}(E)\right) e^{-2 \pi i n j / p}\right)$. So $\mu=$ $\sum_{n=0}^{p-1}\left(1 / p \sum_{j=0}^{p-1} e^{-2 \pi i n j / p} \mu \circ \beta\right)$. The terms of this sum are the claimed formulas. We already know that the $k$ th parts are unique. So all we need to do is check that these terms are of the correct types. So compute

$$
\begin{aligned}
\left(\frac{1}{p} \sum_{j=0}^{p-1} e^{-2 \pi i n j / p} \mu \circ \beta^{j}\right) \circ \beta & =\left(\frac{1}{p} \sum_{j=0}^{p-1} e^{-2 \pi i n j / p} \mu \circ \beta^{j+1}\right) \\
& =\frac{1}{p} \sum_{j=1}^{p} e^{-2 \pi i n(j-1) / p} \mu \circ \beta^{j}
\end{aligned}
$$

(re-index sum; sum is really Haar measure on $\mathbf{Z}_{\mathbf{p}}$ ). So

$$
e^{2 \pi i n / p}\left(\frac{1}{p} \sum_{j=1}^{p} e^{-2 \pi i n j / p} \mu \circ \beta^{j}\right)=e^{2 \pi i n / p}\left(\frac{1}{p} \sum_{j=0}^{p-1} e^{-2 \pi i n j / p} \mu \circ \beta^{j}\right)
$$

(since $e^{2 \pi i p / p} \mu \circ \beta^{p}=e^{2 \pi i 0 / p} \mu \circ \beta^{0}=\mu$ and $\beta^{0} \in(A)$ ). This completes Claim 1 .

Note if $\mu$ is $A$-invariant, each $\mu_{k}$ is $A$-invariant too. This follows since $\mu \circ \beta$ is $A$-invariant when $\mu$ is $A$-invariant.

ClaIM 2. The measure $\mu_{k} * \nu_{l}$ is of type $(k+l) \bmod p$ for $A$-invariant $\mu, \nu \in$ $M(G)$.

ProOF. We have $\left(\mu_{k} * \nu_{l}\right)=\left(\mu_{k} \circ \beta\right) *\left(\nu_{l} \circ \beta\right)=\left(e^{2 \pi i k / p} \mu_{k}\right) *\left(e^{2 \pi i l / p} \nu_{l}\right)=$ $e^{2 \pi i(k+l) / p} \mu_{k} * \nu_{l}$. This completes Claim 2 .

ClaIM 3. We have $f(\sigma)=f\left(\sigma_{0}\right)$ for all $A$-invariant $\sigma \in M(G)$.

PROOF. The function $f$ is $B$-invariant so $(f \circ \beta)\left(\sigma_{k}\right)=f\left(\sigma_{k}\right)$. But $(f \circ \beta)\left(\sigma_{k}\right)=f\left(\sigma_{k} \circ \beta\right)=f\left(e^{2 \pi i k / p} \sigma_{k}\right)=e^{2 \pi i k / p} f\left(\sigma_{k}\right)$. Since $e^{2 \pi i k / p}=1$ if and only if $k=0 \bmod p, f\left(\sigma_{k}\right)=0$ of $k \neq 0 \bmod p$. This completes Claim 3 .

Note that it can be shown that the 0th part of the product of two $A$-invariant measures $\mu, \nu$ can be written as

$$
\mu_{0} * \nu_{0}+\mu_{1} * \nu_{p-1}+\mu_{2} * \nu_{p-2}+\cdots+\mu_{p-1} * \nu_{1} .
$$

(This is just by Claim 2, and uniqueness of the parts.) If $\mu$ is just of type $k$, then the 0th part of $\mu * \nu$ is just $\mu * \nu_{p-k}$. Thus for such $\mu, \nu, f(\mu * \nu)=f\left(\mu * \nu_{p-k}\right)$.

Now to construct the needed measure $\eta$. Suppose there exists an $A$-invariant $\omega \in M(G)$ of type 1 , such that $f\left(\omega^{p}\right)=1$. Then let

$$
\eta=p_{0}+\omega+\omega^{2}+\omega^{3}+\cdots+\omega^{p-1} \text {. }
$$

ClaIM 4. We have $f\left(\eta * \mu_{k} * \nu_{l}\right)=f\left(\eta * \mu_{k}\right) f\left(\eta * \nu_{l}\right)$ for $A$-invariant $\mu, \nu$. 
PROOF. We have

$$
\begin{aligned}
& f\left(\eta * \mu_{k}\right) f\left(\eta * \nu_{l}\right)=f\left(\omega^{p-k} * \mu_{k}\right) f\left(\omega^{p-l} * \nu_{l}\right) \quad \text { (Claim 3) } \\
& =f\left(\omega^{p-k} * \mu_{k} * \omega^{p-l} * \nu_{l}\right)=f\left(\omega^{2 p-k-l} * \mu_{k} * \nu_{l}\right) \\
& = \begin{cases}f\left(\omega^{p}\right) f\left(\omega^{p-k-l} * \mu_{k} * \nu_{l}\right), & k+l<p, \\
f\left(\omega^{2 p-k-l} * \mu_{k} * \nu_{l}\right), & k+l \geq p,\end{cases} \\
& = \begin{cases}f\left(\omega^{p-k-l} * \mu_{k} * \nu_{l}\right), & k+l<p, \\
f\left(\omega^{2 p-k-l} * \mu_{k} * \nu_{l}\right), & k+l \geq p,\end{cases} \\
& =f\left(\eta * \mu_{k} * \nu_{l}\right) \quad(\text { Claim 3). }
\end{aligned}
$$

So Claim 4 is done.

ClaIm 5. The functional $f_{\eta}$ is multiplicative on $A$-invariant measures.

ProOF. We compute

$$
\begin{aligned}
f(\eta * \mu * \nu) & =\sum_{k=0}^{p-1} \sum_{l=0}^{p-1} f\left(\eta * \mu_{k} * \nu_{l}\right) \\
& =\sum_{k=0}^{p-1} \sum_{l=0}^{p-1} f\left(\eta * \mu_{k}\right) f\left(\eta * \nu_{l}\right) \quad \text { (Claim 4) } \\
& =\left(\sum_{k=0}^{p-1} f\left(\eta * \mu_{k}\right)\right)\left(\sum_{l=0}^{p-1} f\left(\eta * \nu_{l}\right)\right) \\
& =f(\eta * \mu) f(\eta * \nu) .
\end{aligned}
$$

So Claim 5 is done.

Claim 6. The functional $f_{\eta}$ is $A$-invariant.

Proof. Pick $\sigma \in M(G)$. We want to show $f_{\eta}(\sigma)=f_{\eta}(\sigma \circ \alpha)$ for all $\alpha \in A$. So $f_{\eta}(\sigma \circ \alpha)=f(\eta *(\alpha \circ \alpha) \beta)=f\left(\left(\left(\eta \circ \alpha^{-1}\right) * \sigma\right) \circ \alpha\right)=f\left(\left(\eta \circ \alpha^{-1}\right) * \sigma\right)(f$ is $B$-invariant $)=f(\eta * \sigma)=f_{\eta}(\sigma)(f$ is $B$-invariant and $\eta$ is $A$-invariant $)$. So Claim 6 is done.

Claim 7. If no $\omega$ of type 1 can be found such that $f\left(\omega^{p}\right)=1$, then $\eta=$ $p_{0}$ suffices, i.e., $f$ is already multiplicative on $A$-invariant measures and is $A$ invariant.

ProOF. If there is no $\omega$ of type 1 such that $f\left(\omega^{p}\right)=1$, then $f\left(\xi^{p}\right)=0$ for all $\xi \in M(G)$ of type 1. (If $f\left(\xi^{p}\right) \neq 0$ for some $\xi \in M(G)$ of type 1 , then $\xi$ could be rescaled to force $f\left(\xi^{p}\right)=1$.)

We will show that $f\left(\mu_{k} * \nu_{l}\right)=0$ if $k \neq 0$ or $l \neq 0$, for $A$-invariant $\mu, \nu \in M(G)$. Without loss of generality $k \neq 0$. If $(k+l) \neq 0 \bmod p$, then $f\left(\mu_{k} * \nu_{l}\right)=0$ already by Claim 3. Otherwise $(k+l)=0 \bmod p$; select $j>0$ such that $j k=1 \bmod p$. Then $f\left(\mu_{k} * \nu_{l}\right)^{j p}=f\left(\left(\mu_{k} * \nu_{l}\right)^{j p}\right)=f\left(\left(\mu_{k}\right)^{j p} *\left(\nu_{l}\right)^{j p}\right)=f\left(\left(\mu_{k}\right)^{j p}\right) f\left(\left(\nu_{l}\right)^{j p}\right)$ 
(since $\left(\mu_{k}\right)^{j p}$ and $\left(\nu_{l}\right)^{j p}$ are of type 0$)=0\left(\right.$ since $\left(\mu_{k}\right)^{j p}=\left(\mu_{k}^{j}\right)^{p}$ is a measure of type 1 to the $p$ th power). So $f\left(\mu_{k} * \nu_{l}\right)=0$.

Then $f(\mu * \nu)=\sum_{k=0}^{p-1} \sum_{l=0}^{p-1} f\left(\mu_{k} * \nu_{l}\right)=f\left(\mu_{0} * \nu_{0}\right)=f\left(\mu_{0}\right) f\left(\nu_{0}\right)=f(\mu) f(\nu)$ (Claim 3).

This completes Claim 7, and the proof of the theorem.

COROLLARY 5.4. Let $B$ be a solvable finite group of automorphisms on a locally compact abelian group $G$. Then if $f \in M(G)^{*}$ is $B$-invariant and multiplicative on $B$-invariant measures, there exists $\eta \in M(G)$ such that $f_{\mu} \in \Delta M(G)$.

Proof. Use the theorem and induction; $B$ solvable means $B$ can be written

$$
\{e\} \triangleleft B_{1} \triangleleft B_{2} \triangleleft \cdots \triangleleft B_{n-1} \triangleleft B_{n}=B
$$

where $B_{k} / B_{k-1}=\mathbf{Z}_{q(k)}$ for some prime $q(k)$, for $k=1,2, \ldots, n$. (See Hungerford $[8$, II8.6].)

With the preceding theorem, it becomes easy to show that $M(G)_{B}$ is structure-strong. In fact $\Delta\left(M(G)_{B}\right)$ spans an algebra (even before the closure is taken).

For $f \in M(G)^{*}$ define $f_{B} \in M(G)^{*}$ to be $f_{B}=\frac{1}{n} \sum_{\beta \in B} f \circ \beta$. (Recall $n=|B|$.) Easily $f_{B}$ is $B$-invariant.

LEMMA 5.5. Suppose $f, g \in \Delta M(G)$. Then $f_{B} g_{B}=\frac{1}{n} \sum_{\beta \in B}[(f)(g \circ \beta)]_{B}$.

Proof. We have

$$
\begin{aligned}
\frac{1}{n} \sum_{\beta \in B}[(f)(g \circ \beta)]_{B} & =\frac{1}{n} \sum_{\beta \in B} \frac{1}{n} \sum_{\delta \in B}[(f)(g \circ \beta)] \circ \delta \\
& =\frac{1}{n} \sum_{\beta \in B} \frac{1}{n} \sum_{\delta \in B}(f \circ \delta)(g \circ \beta \delta) \\
& =\frac{1}{n} \sum_{\delta \in B}(f \circ \delta)\left(\frac{1}{n} \sum_{\beta \in B} g \circ \beta \delta\right) \\
& =\left(\frac{1}{n} \sum_{\delta \in B} f \circ \delta\right)\left(\frac{1}{n} \sum_{\beta \in B} g \circ \beta\right)=f_{B} g_{B}
\end{aligned}
$$

using Lemma 4.4 .

THEOREM 5.6. $M\left(G_{B}\right)$ (or $\left.M(G)_{B}\right)$ is structure-strong. In fact, if $f, g \in$ $\Delta\left(M(G)_{B}\right)$ then there exists $k_{1}, k_{2}, \ldots, k_{n} \in \Delta\left(M(G)_{B}\right)$ such that

$$
f g=(1 / n)\left(k_{1}+k_{2}+\cdots+k_{n}\right) \text {. }
$$


PROOF. For $f, g \in \Delta\left(M(G)_{B}\right)$, there exists $h, j \in \Delta M(G)$ such that $f=h_{B}$, $g=j_{B}$ (there exists $h, j \in \Delta M(G)$ such that $h=f, g=j$ when restricted to $M(G)_{B}$; easily $f=h_{B}, g=j_{B}$ on all of $M(G)$, since $h_{B}, j_{B}$ obey $h_{B}(\mu)=h\left(\mu_{B}\right)$, $j_{B}(\mu)=j\left(\mu_{B}\right)$ for all $\mu \in M(G)$.) Then

$$
f g=h_{B} j_{B}=\frac{1}{n} \sum_{\beta \in B}[(f)(g \circ \beta)]_{B}
$$

by the previous lemma. Recall if $j \in \Delta M(G)$ then $j \circ \beta \in \Delta M(G)$. So by Taylor [17, 3.2.3], $(h)(j \circ \beta) \in \Delta M(G)$. Then $[(h)(j \circ \beta)]_{B} \in \Delta\left(M(G)_{B}\right)$ for each $\beta \in B$. So the $n$ different functionals $[(h)(j \circ \beta)]_{B}$ (for $\beta \in B$ ) form the functionals $k_{1}, k_{2}, \ldots, k_{n}$ of the theorem.

Now for $f \in \Delta\left(M(G)_{B}\right)$, consider $f^{*} \in M(G)^{*}$, the involution of $f$ defined by $f^{*}(\mu)=f(\bar{\mu})^{-}$for all $\mu \in M(G)$. Then $f^{*} \in \Delta\left(M(G)_{B}\right)$ : For $\mu, \nu \in M(G)_{B}$, $f^{*}(\mu * \nu)=f\left((\mu * \nu)^{-}\right)^{-}=f(\bar{\mu} * \bar{\nu})^{-}=(f(\bar{\mu}) f(\bar{\nu}))^{-}=\left[f(\bar{\mu})^{-}\right]\left[f(\bar{\nu})^{-}\right]$.

Thus $\Delta\left(M(G)_{B}\right)$ spans a $*$-subalgebra of the commutative unital $B^{*}$-algebra $M(G)^{*}$. So the closure of the span is a commutative unital $B^{*}$-algebra. Thus $M(G)_{B}=M\left(G_{B}\right)$ is structure-strong.

Now all the results for structure-strong hypergroup measure algebras apply: There is a structure semihypergroup $K$ as in Theorem 4.1. But in fact a better description of the semihypergroup is possible. We will show that if $S$ is the structure semigroup of $M(G)$ then $K$ is equal to the space of $B$-orbits of $S$, defined in a natural way.

DEFINITIONS. Let $A \subseteq M(G)^{*}$ be the norm-closure of the linear span of $\Delta M(G)$. Recall $S=\Delta A$ is the structure semigroup of $M(G)$ (Taylor [17, 3.2.3]). For $F \in A^{*}$ define $F \circ \beta \in A^{*}$ by $(F \circ \beta)(f)=F(f \circ \beta)$ for all $f \in A$. In particular, if $s \in S=\Delta A \subseteq A^{*}$, define $s \circ \beta \in S$ by $s \circ \beta(f)=s(f \circ \beta)$ for all $f \in A$. (We have $s \circ \beta \in S$ since $(s \circ \beta)(f g)=s((f g) \circ \beta)=s((f \circ \beta)(g \circ \beta)=s(f \circ \beta) s(g \circ \beta)=$ $s \circ \beta(f) s \circ \beta(g)$.) Define $s_{B} \in A^{*}$ for $s \in S$ by $\frac{1}{n} \sum_{\beta \in B} s \circ \beta$. Define $S_{B} \subseteq A^{*}$ to be $\left\{s_{B}: s \in S\right\}$.

Proposition 5.7. Consider $M(G)_{B} \subseteq M(G)$. Let $K$ be the structure semihypergroup of $M(G)_{B}$. Then if $S$ is the structure semigroup of $M(G)$, it follows that $K=S_{B}$.

Proof. Recall $A=$ closed linear span of $\Delta M(G)$ and $S=\Delta A$. We have shown that $\Delta\left(M(G)_{B}\right)=\Delta M(G)$ restricted to $M(G)_{B}$. In fact, $\Delta\left(M(G)_{B}\right)=$ $(\Delta M(G))_{B}=\left\{f_{B}: f \in \Delta M(G)\right\}$. (Recall $f_{B}=\frac{1}{n} \sum_{\beta \in B} f \circ \beta$.) (Note $f$ restricted to $M(G)_{B}$ agrees with $f_{B}$ on $M(G)_{B}$; easily $f_{B}(\mu)=f\left(\mu_{B}\right)$.) Then the closed linear span of $\Delta\left(M(G)_{B}\right)$ is $A_{B}=\left\{f_{B}: f \in A\right\}$. Recall $K=\Delta\left(A_{B}\right)$. In fact, $K=\Delta\left(A_{B}\right)=(\Delta A)_{B}=S_{B}$. The claim is that $\Delta\left(A_{B}\right)=\Delta A$ restricted to $A_{B} \subseteq A$. This can be shown in exactly the same was as Theorem 5.3. (Note 
$(\Delta A)_{B}=\Delta A$ restricted to $A_{B}$, in the same way $\Delta\left(M(G)_{B}\right)=(\Delta M(G))_{B}$ : that is, $s_{B}$ agrees with $s$ on $A_{B}$.)

THEOREM 5.8. The space $S_{B}$ is a semihypergroup in a natural way and the product agrees with that of $K$.

Proof. Let $s, t \in S$. Then $s_{B} t_{B}=\frac{1}{n} \sum_{\beta \in B}[s(t \circ \beta)]_{B}$. This is shown precisely as in Proposition 5.5, except it must be verified that $(s t) \circ \beta=$ $(s \circ \beta)(t \circ \beta)$ for all $\beta \in B$ (note $s \circ \beta \in S$ if $s \in S$ ). Here we must work with the Arens product:

$$
\begin{array}{ll}
\langle f, \mu\rangle(\nu)=f(\mu * \nu) & \text { for all } \mu, \nu \in M(G), f \in A ; \\
{[t, f](\mu)=t(\langle f, \mu\rangle)} & \text { for all } \mu \in M(G), f \in A, t \in S ; \\
s t(f)=s([t, f]) & \text { for all } f \in A, s, t \in S \subseteq A^{*} .
\end{array}
$$

Note $[t, f] \in A$ and $\langle f, \mu\rangle \in A$; argue as in Theorem 4.1.

Let $\beta \in B, f \in A$, and $\mu, \nu \in M(G)$. Then $\langle f \circ \beta, \mu\rangle(\nu)=(f \circ \beta)(\mu * \nu)=$ $f((\mu * \nu) \circ \beta)=f((\mu \circ \beta) *(\nu \circ \beta))=\langle f, \mu \circ \beta\rangle(\nu \circ \beta)$. Thus $\langle f \circ \beta, \mu\rangle=\langle f, \mu \circ \beta\rangle \circ \beta$.

Now let $t \in S$. Then $[t, f \circ \beta](\mu)=t(\langle f \circ \beta, \mu\rangle)=f(\langle f, \mu \circ \beta\rangle \circ \beta)=$ $t \circ \beta(\langle f, \mu \circ \beta\rangle)=[t \circ \beta, f](\mu \circ \beta)$. Thus $[t, f \circ \beta]=[t \circ \beta, f] \circ \beta$.

For $s \in S,((s t) \circ \beta)(f)=(s t)(f \circ \beta)=s([t, f \circ \beta])=s([t \circ \beta, f] \circ \beta)=$ $s \circ \beta([t \circ \beta, f])=(s \circ \beta)(t \circ \beta)(f)$. Thus $(s t) \circ \beta=(s \circ \beta)(t \circ \beta)$ as claimed.

To see that the products of $S_{B}$ and $K$ agree, note that $S_{B} \subseteq A^{*}$ gets its product from $A^{*}$ (constructed by Rennison using the Arens product), and $K=$ $\Delta\left(A_{B}\right) \subseteq\left(A_{B}\right)^{*} \subseteq A^{*}$ gets its product in effectively the same way, as constructed in the proof of Theorem 4.1. Finally, we may identify $K$ with the set of $B$-orbits of $S,\{\{\beta(s): \beta \in B\}: s \in S\}$, where $\beta(s)=s \circ \beta$. This is because $\beta(s)=s \circ \beta$ agrees with $s$ on $A_{B}$, and also agrees with $s_{B}=\frac{1}{n} \sum_{\beta \in B} s \circ \beta$. (Note that if $s \circ \beta=t \circ \delta$ on $A_{B}$ for $s, t \in S, \beta, \delta \in B$, then $s=s \circ \beta$ and $t=t \circ \delta$ must agree on $A_{B}$.)

REMARK. In the next section, hypergroup measure algebras first studied by Ragozin will be shown to be structure-strong. These examples arise from the orbit spaces of certain infinite groups of automorphisms. It seems natural to attempt to generalize the preceding example to infinite groups $B$, say $B$ an infinite cartesian product of finite groups. However, difficulties arise, which make it unclear whether such algebras are structure-strong. For example, let $G=$ $\prod_{i=0}^{\infty} T=T^{\omega}$ and let $B=\{-1,1\}^{\omega}$ (so $\beta \in B$ is of the form $\beta\left(\left(t_{1}, t_{2}, t_{3}, \ldots\right)\right.$ ) $=$ $\left(\beta_{1} t_{1}, \beta_{2} t_{2}, \beta_{3} t_{3}, \ldots\right)$ where $\beta_{i} t_{i}=t_{i}$ or $\left.-t_{i}\right)$. (Here we identify $T$ with $[-\pi, \pi]$.) Then it may be shown that $G$ and $B$ are compact abelian groups, each $\beta \in B$ is a continuous automorphism, and the action $(\beta, g) \rightarrow \beta(g): B \times G P \rightarrow G$ is continuous. So $G_{B}$ is a hypergroup (Jewett $[9$, Theorem $8.3 \mathrm{~A}]$ ). Following the program of this section, the first goal would be to locate, for $f \in \Delta M_{B}(G)$, an 
$f^{\prime} \in \Delta M(G)$ such that $f^{\prime}=f$ on $M_{B}(G)$. It is unclear if such an $f^{\prime}$ is available. But there is an $L$-subalgebra $\mathscr{B}$ of $M(G)$ containing $M_{B}(G)$ such that there exists $f^{\prime} \in \Delta \mathscr{B}$ with $f^{\prime}=f$ on $M_{B}(G)$. This algebra is simply the smallest $L$ algebra containing $M_{B}(G)$, and may be characterized by $\mathscr{B}=\{\mu \in M(G) \mid \beta \rightarrow$ $\mu \circ \beta: B \rightarrow M(G)$ is norm continuous $\}. \mathscr{B}$ is structure-strong (it satisfies the definition of Taylor [17] of a commutative convolution measure algebra), so $\triangle \mathscr{B} \cup\{0\}$ is a semigroup. The next step of the program of this section would be to produce a suitable analogue of Lemma 5.5 , for $M_{B}(G) \subseteq \mathscr{B}$. Then Theorem 6 would follow from Lemma 5.5 using $\triangle \mathscr{B} \cup\{0\}$ being a semigroup. Lemma 5.5 is the source of the difficulty. The sums of Lemma 5.5 turn into vector integrals. The map $\beta \rightarrow f \circ \beta: B \rightarrow \Delta \mathscr{B} \subseteq \mathscr{B}^{*}$ is not known to be measurable. Thus the integrals are not known to be defined, and the issue of whether $f g$ is normapproximable by members of $\Delta M_{B}(G)$ (for $f, g \in \Delta M_{B}(G)$ ) is still unresolved.

\section{Other examples of structure-strong hypergroup measure algebras}

Here two examples will be considered, which were studied by D. Ragozin [11, 12].

Let $G$ be a connected compact simple Lie group, for example, SU(2). Using the characterization of $\Delta Z M(G)$ due to D. Ragozin [11] (where $Z M(G) \subseteq M(G)$ is the subalgebra of central measures on $G$ ), it may be shown that $Z M(G)$ is structure-strong.

Let $\Sigma$ be the set of equivalence classes of irreducible unitary representations of $G$. Let $I$ be the group of inner automorphisms on $G$. Let $G_{I}$ be the space of $I$ orbits of $G$ (that is, conjugacy classes). Then $Z M(G)$ and $M\left(G_{I}\right)$ are isomorphic as Banach algebras. Let $Z$ be the center of $G$. (Note the center of a connected compact simple Lie group is finite.)

Ragozin showed that $\Delta Z M(G)=\Delta M\left(G_{I}\right)$ is given by $\Sigma \cup Z$. He gave the explicit formulas: $\phi \in \Sigma$ produces the multiplicative linear functional on $Z M(G)$ given by $\phi(\mu)=1 / d_{\phi} \int_{G} \chi_{\phi}\left(g^{-1}\right) d \mu(g)$ for all $\mu \in Z M(G)$, where $\chi_{\phi}$ is the representation character of $\phi$ (and $\phi$ has dimension $d_{\phi}$ ). Also $\gamma \in Z^{\wedge}$ produces the multiplicative linear functional on $Z M(G)$ given by $\gamma(\mu)=\int_{G} \bar{\gamma}\left(g^{-1}\right) d \mu(g)$ for all $\mu \in Z M(G)$, where $\bar{\gamma}$ is defined by

$$
\bar{\gamma}(g)= \begin{cases}\gamma(g) & \text { if } g \in Z \\ 0 & \text { if } g \notin Z .\end{cases}
$$

Ragozin shows that if $\mu \in Z M(G)$ is continuous, then $\gamma(\mu)=0$, while if $\mu$ is discrete (supported on $Z$ ), then $\phi(\mu)=0$.

To consider the product of $M\left(G_{I}\right)^{*}=Z M(G)^{*}$, we use the following lemma (discussed in Rennison [13, 2.2]). 
LEMMA 6.1. Let $H$ be a locally compact Hausdorff space. Then the product in Taylor $[17,2.3]$ (of Sreider generalized functionals) on $M(H)^{*}$ agrees with the Arens product on $M(H)^{*}=C_{0}(H)^{* *}$.

PrOOF. The Arens product on $C_{0}(H)^{* *}$ is given by

(1) $\langle\mu, \rho\rangle(\tau)=\int_{H} \rho \tau d \mu$ for all $\rho, \tau \in C_{0}(H), \mu \in C_{0}(H)^{*}=M(H)$;

(2) $[f, \mu](\rho)=f(\langle\mu, \rho\rangle)$ for all $\rho \in C_{0}(H), \mu \in C_{0}(H)^{*}, f \in C_{0}(H)^{* *}$;

(3) $(f g)(\mu)=f([g, \mu])$ for all $f, g \in C_{0}(H)^{* *}, \mu \in C_{0}(H)^{*}$.

Recall the Sreider product is given by: for $f \in M(H)^{*}, \mu \in M(H), \nu \in M(H)$ with $\nu \ll \mu$, we have $f(\nu)=\int_{H} f_{\mu} d \nu$, where $f_{\mu} \in L^{\infty}(H, \mu)$ is given by $f$ restricted to $L^{1}(H, \mu)$. Then if $f, g \in M(H)^{*}$, let $f g(\mu)=\int_{H} f_{\mu} g_{\mu} d \mu$.

Now if $\mu \in M(H), f \in M(H)^{*}, \rho \in C_{0}(H)$, then $[f, \mu](\rho)=f(\langle\mu, \rho\rangle)=$ $\int_{H} f_{\mu} \rho d \mu$. This is because $\langle\mu, \rho\rangle \ll \mu$ (consider (1) above). So $d[f, \mu]=f_{\mu} d \mu$. Then for $f, g \in M(H)^{*}, \mu \in M(H),(f, g)(\mu)=f([g, \mu])=\int_{H} f_{\mu} d[g, \mu]=$ $\int_{H} f_{\mu} g_{\mu} d \mu$.

This lemma enables us to consider the Arens product on $Z M(G)^{*} \subseteq M(G)^{*}$, instead of the Sreider product on $M\left(G_{I}\right)^{*}$.

THEOREM 2. If $\phi, \psi \in \Delta Z M(G)$ then one of the following must occur:

(1) $\phi, \psi \in \Sigma$, in which case $\phi \psi$ is a convex combination of multiplicative linear functionals from $\triangle Z M(G)$

(2) $\phi, \psi \in Z^{\wedge}$, in which case $\phi \psi \in Z^{\wedge}$ is again a multiplicative linear functional from $\triangle Z M(G)$

(3) $\phi \in \Sigma, \psi \in Z^{\wedge}$ (or vice-versa), in which case $\phi \psi=0$. Thus $\Delta Z M(G)$ spans a commutative unital $B^{*}$-algebra.

PROOF. (1) If $\phi, \psi \in \Sigma$ then $\phi \psi$ is given by integration against

$$
\left(1 / d_{\phi} \chi_{\phi}\right)\left(1 / d_{\psi} \chi_{\psi}\right)
$$

which is a convex combination of representation characters (see, for example, Jewett $[9,8.4 \mathrm{~B}])$.

(2) $\phi \psi \in Z^{\wedge}$ is clear; they are just group characters. So $\phi \psi(\mu)=\int_{G} \bar{\phi} \psi^{-} d \mu$ for all $\mu \in Z M(G)$ is multiplicative linear functional.

(3) We consider $Z M(G)^{*}$ with the Arens product. Now $Z M(G)=Z M_{d}(G) \oplus$ $Z M_{c}(G)$ where $Z M_{d}(G)$ is the set of discrete central measures and $Z M_{c}(G)$ is the set of continuous central measures. Suppose $\phi \in \Sigma$ and $\gamma \in Z^{\wedge}$. Then $\phi(\mu)=0$ if $\mu \in Z M_{d}(G)$ and $\gamma(\mu)=0$ if $\mu \in Z M_{c}(G)$. Suppose $\mu \in Z M_{c}(G)$. Then $[\gamma, \mu]=0:[\gamma, \mu](\tau)=\gamma(\langle\mu, \tau\rangle)=0$ for all $\tau \in C_{0}(G)$ since $\langle\mu, \tau\rangle$ is continuous $\left(\langle\mu, \tau\rangle(\rho)=\int_{G} \rho \tau d \mu\right.$ so $\left.\langle\mu, \tau\rangle \ll \mu\right)$. Then $\phi \gamma(\mu)=\phi([\gamma, \mu])=\phi(0)=0$. Now consider $\mu \in Z M_{d}(G)$. The space $Z M(G)^{*}=M\left(G_{I}\right)^{*}$ has a commutative 
product (see proof of previous lemma), so $\phi \gamma=\gamma \phi$. Then $\phi \gamma(\mu)=\gamma \phi(\mu)=$ $\gamma([\phi, \mu])=0$ since $[\phi, \mu]=0\left([\phi, \mu](\tau)=\phi(\langle\mu, \tau\rangle)=0\right.$ for all $\tau \in Z C_{0}(G)$ since $\langle\mu, \tau\rangle \ll \mu$ is discrete). Thus $\phi \gamma=0$.

Now it is clear that $\Delta Z M(G)$ spans a subalgebra of $Z M(G)^{*}=M\left(G_{I}\right)^{*}$. The norm-closure of the span will be Banach algebra. In fact, since $\chi_{\pi}^{-}$is $\chi_{\bar{\pi}}$ where $\bar{\pi}$ is the conjugate representation of $\pi \in \Sigma$ (see Hewitt and Ross [7, volume II, 27.27]), and since $\bar{\gamma} \in Z^{\wedge}$ for $\gamma \in Z^{\wedge}$, we see this algebra is a $B^{*}$-algebra. Thus $Z M(G)=M\left(G_{I}\right)$ is a structure-strong hypergroup measure algebra.

The results of Section 5 effectively deal with hypergroups of measures on $R^{2}$ invariant under finite groups of rotations. (Such groups of rotations are of course finite solvable groups.) Here we show that the hypergroup measure algebra $M\left(R^{2}\right)_{T}$ of measures invariant under the group $T$ of all rotations around the origin is structure-strong. The difficulty here is that $T$ is not finite; those techniques do not apply. However, the results of D. Ragozin [12] do apply.

\section{THEOREM 6.3. $M\left(R_{T}^{2}\right)=M\left(R^{2}\right)_{T}$ is structure-strong.}

ProOF. Ragozin showed that $\Delta\left(M\left(R^{2}\right)_{T}\right)=\{\infty\} \cup\left(R_{T}^{2}\right)^{\wedge}$, where $\left(R_{T}^{2}\right)^{\wedge}$ is the usual set of hypergroup characters, and the point at infinity is the multiplicative linear functional $\mu \rightarrow \mu(0): M\left(R^{2}\right)_{T} \rightarrow C$. The hypergroup characters on $R_{T}^{2}$ identified with $[0, \infty)$ are of the form $J(c x)$ for $c \geq 0$, where $J$ is the Bessel function of order 0 . (See Jewett $[9,9.3]$ ). As usual, the characters induce multiplicative linear functionals by integration.

The product of the hypergroup characters is given by (for $a, b \geq 0$ ),

$$
J(a x) J(b x)=\frac{1}{2 \pi} \int_{0}^{2 \pi} J(c(t) x) d t
$$

where $c(t)=\left(a^{2}+b^{2}-2 a b \cos t\right)^{1 / 2}$ (see Jewett [9, 9.3]). Since $c$ is continuous and $J \in C_{0}([0, \infty))$, it is clear that the pointwise product of two characters may be uniformly approximated by sums of characters. Therefore the closure under the uniform norm of the linear span of $\left(R_{T}^{2}\right)^{\wedge}$ is a Banach algebra. The norm matches the norm of $M\left(R_{T}^{2}\right)^{*}$. Since the characters are real-valued, the Banach algebra they span is a $B^{*}$-algebra, where involution is just complex conjugation, and the unit is $J(0 x) \equiv 1$.

Note that Hartmann, Henrichs, and Lasser [6], show a more general result that $\left(G_{B}\right)^{\wedge}$ is a hypergroup, where $G$ is a locally compact group and $B$ is a group of automorphisms of $G$ which contains the inner automorphisms, and is relatively compact with respect to the Birkhoff topology.

Now we show the following: If $f_{\infty}(\mu)=\mu(0)$ for all $\mu \in M\left(R^{2}\right)_{T}$ is the point at infinity, and $g \in\left(R_{T}^{2}\right)^{\wedge}$, then $\left(f_{\infty}\right)(g)=0$. By Ragozin [12], if $\mu \in M\left(R^{2}\right)_{T}$ then $\mu=\alpha p_{0}+\mu_{\mathrm{c}}$ where $\mu_{\mathrm{c}}$ is a continuous measure, $p_{0}$ is the point mass at 
the origin, and $\alpha \in \mathbb{C}$. If $\mu=\mu_{c}$, then $f_{\infty}(\mu)=0$. Also $g\left(\alpha p_{0}\right)=0$. Now we may use the Arens product as in Theorem 6.2, with the algebra $C\left(R^{2}\right)_{T}$ and its second dual $M\left(R^{2}\right)_{T}$. Then $\left(f_{\infty} g\right)(\mu)=f_{\infty}([g, \mu])=0$ since easily (as in the proof of Theorem 6.2), $[g, \mu] \ll \mu$ is a continuous measure. Also $\left(f_{\infty} g\right)\left(\alpha p_{0}\right)=$ $\left(g f_{\infty}\right)\left(\alpha p_{0}\right)=g\left(\left[f_{\infty}, \alpha p_{0}\right]\right)=0$ since $\left[f_{\infty}, \alpha p_{0}\right] \ll \alpha p_{0}$ is supported on the origin. Thus $f_{\infty} g=0$.

Finally, we show that $f_{\infty} f_{\infty}=f_{\infty}$. Now

$$
\begin{aligned}
f_{\infty} f_{\infty}(\mu) & =f_{\infty}\left(\left[f_{\infty}, \mu\right]\right)=\left[f_{\infty}, \mu\right](\{0\}) \\
& =f_{\infty}\left(\left.\mu\right|_{\{0\}}\right)=\mu(\{0\})
\end{aligned}
$$

since for all $\rho \in C_{0}\left(R^{2}\right)_{T},\left[f_{\infty}, \mu\right](\rho)=f_{\infty}(\langle\mu, \rho\rangle)=f_{\infty}(\rho d \mu)=\int_{\{0\}} \rho d \mu$. (Consider $\rho$ which approximates $p_{0}$.)

Thus $\Delta M\left(R_{T}^{2}\right)$ spans a commutative unital $B^{*}$-algebra, and $M\left(R_{T}^{2}\right)$ is a structure-strong measure algebra.

\section{References}

[1] P. Civin and B. Yood, 'The second conjugate space of a Banach algebra as an algebra', Pacific J. Math. 12 (1961), 847-870.

[2] J. Duncan and S. Hosseiniun, 'The second dual of a Banach algebra', Proc. Roy. Soc. Edinburgh 84A (1979), 309-325.

[3] C. Dunkl, 'Structure hypergroups for measure algebras', Pacific J. Math. 47 (1973), 413425.

[4] C. Dunkl, 'The measure algebra of a locally compact hypergroup', Trans. Amer. Math. Soc. 179 (1973), 331-348.

[5] S. Grosser and M. Moskowitz, 'Compactness conditions in topological groups', J. Reine Angew. Math. 246 (1971), 1-40.

[6] K. Hartmann, R. W. Henrichs, and R. Lasser, 'Duals of orbit spaces in groups with relatively compact inner automorphism groups are hypergroups', Monatsh Math. 88 (1979), 229-238.

[7] E. Hewitt and K. A. Ross, Abstract harmonic analysis. I, II (Springer-Verlag, New York, 1963,1970 ).

[8] T. Hungerford, Algebra (Graduate Texts in Mathematics, 73, Springer-Verlag, New York, 1974).

[9] R. Jewett, 'Spaces with an abstract convolution of measures', Adv. in Math. 18 (1975), 1-101.

[10] R. Lasser, 'Almost periodic functions on hypergroups', Math. Ann. 252 (1980), 183-196.

[11] D. Ragozin, 'Central measures on compact simple Lie groups', J. Funct. Anal. 10 (1972), 212-229.

[12] D. Ragozin, 'Rotation invariant measure algebras on Euclidean space', Indiana Univ. Math. J. 23 (1974), 1139-1154.

[13] J. Rennison, 'Arens products and measure algebras', J. London Math. Soc. 44 (1969), 369-377.

[14] K. A. Ross, Hypergroups and centers of measure algebras (Istituto Nazionale di Alta Matematica Symposia Mathematica, 22, 1977). 
[15] W. Rudin, Real and complex analysis (2nd ed., McGraw-Hill, 1974).

[16] J. Sreider, 'The structure of maximal ideals in rings of measures with convolution', Mat. Sb. 27 (69) (1950), 297-318; English translation, Amer. Math. Soc. Transl. (1) 8 (1962), 365-391.

[17] J. L. Taylor, Measure algebras (CBMS Regional Conf. Ser. in Math., no. 16, Amer. Math. Soc., Providence, R. I., 1973).

Department of Mathematics

and Statistics

Mississippi State University

Mississippi State

Mississippi 39762

U.S.A. 